\title{
MicroRNA expression and its detection in human supraglottic laryngeal squamous cell carcinoma
}

\author{
XIN SUN ${ }^{1}$, YAN SONG ${ }^{1}$, XUHUI TAI ${ }^{2}$, BIN LIU $^{1}$ and WENYUE JI ${ }^{1}$ \\ ${ }^{1}$ Department of Otorhinolaryngology, Shengjing Hospital, China Medical University, Shenyang, Liaoning 110004; \\ ${ }^{2}$ Department of Otorhinolaryngology, Chinese PLA 463 Hospital, Shenyang, Liaoning 110042, P.R. China
}

Received May 14, 2013; Accepted July 10, 2013

DOI: $10.3892 /$ br.2013.143

\begin{abstract}
Laryngeal squamous cell carcinoma (LSCC) is one of the most common head and neck malignancies with a poor prognosis. The aim of this study was to detect the expression of microRNAs (miRNAs) in supraglottic LSCC and attached normal mucosa using microarray assay, and explore the role of miRNAs in supraglottic LSCC. Fresh-frozen samples of five supraglottic LSCC and attached normal mucosa were obtained, and their various endogenous miRNAs were screened by microarray. miRNAs of interest were confirmed by quantitative real-time polymerase chain reaction (qRT-PCR) in 48 supraglottic LSCC tumors and 10 normal mucosa tissues. In total, 38 miRNAs were differentially expressed in supraglottic LSCC, with 22 miRNAs being upregulated and 16 downregulated. Of the six miRNAs selected for qRT-PCR, miR-375, miR-139-3P, miR-1290 and miR-106b were differentially expressed in supraglottic LSCC with and without lymphatic metastasis.
\end{abstract}

\section{Introduction}

Laryngeal squamous cell carcinoma (LSCC) is one of the most common head and neck malignancies and has been reported to account for $\sim 2.4 \%$ of all new malignancies worldwide each year (1). The prognosis for LSCC has not shown any improvement in the last 30 years (2), due to lymphatic metastasis. Additionally, the supraglottic type frequently undergoes lymphatic metastasis in LSCC. A better understanding of the molecular pathways that result in lymphatic metastasis of supraglottic LSCC is essential in the identification of novel molecular biomarkers which have clinical utility in predicting prognosis and therapeutic efficacy, as well as in designing targeted therapy for this disease.

Correspondence to: $\mathrm{Dr}$ Wenyue Ji, Department of Otorhinolaryngology, Shengjing Hospital, China Medical University, 36 Sanhao Street, Heping, Shenyang, Liaoning 110004, P.R. China E-mail: ji-wenyue@hotmail.com

Key words: microarray, quantitative real-time polymerase chain reaction, supraglottic laryngeal carcinoma, lymphatic metastasis
MicroRNAs (miRNAs) are a class of short non-coding RNAs that modulate gene expression by targeting mRNAs and triggering either the repression of translation or RNA degradation (3). Due to the partial complementarity between miRNAs and their target transcripts, a single miRNA is capable of simultaneously regulating hundreds of genes, and therefore carries a significant modulatory potential (4). miRNAs are involved in tissue differentiation during both physiological and pathological processes (5). Previous studies on different cancer types have identified the emergence of distinct miRNA expression profiles between tumor tissues and their corresponding normal tissues $(6,7)$. Another study identified miRNA expression profiles capable of distinguishing the different tumor subtypes or developmental lineages (8). Increasing data support the value of miRNA expression profiles as a novel biomarker in diagnosis, prognosis and as a new target in therapy.

In this study, the miRNA expression of normal laryngeal epithelia was compared with primary human supraglottic LSCC at advanced stage to define those miRNA that are most capable of differentiating disease, thus having the greatest potential as biomarkers and therapy targets.

\section{Materials and methods}

Tissue samples. Five pairs of tumor and adjacent normal epithelial tissues were obtained from supraglottic LSCC patients undergoing total laryngectomy in the Shengjing Hospital, China Medical University in January, 2012. Tissue samples were used for microarray analysis. Another 48 patients with supraglottic LSCC, who were treated at the Department of Otorhinolaryngology, Shengjing Hospital, China Medical University, from July, 2011 to November, 2012, were included for qRT-PCR. All the patients were diagnosed pathologically to be supraglottic LSCC and received no radiation and chemotherapy preoperatively. All the patients provided informed consent preoperatively. The study was approved by the ethics committee of the Shengjing Hospital of China Medical University. Ten patients underwent total laryngectomy and 38 underwent partial laryngectomy (except surgery by laser), 17 patients had while 31 did not have lymphatic metastasis. Samples were immediately snap-frozen in liquid nitrogen and stored at $-80^{\circ} \mathrm{C}$. Mucosas, which were obtained from 10 patients with total laryngectomy and were $>2.0 \mathrm{~cm}$ away 
from the tumor margin, were used as the control. The mucosas were histologically normal. TNM classification definitions were according to the UICC (2002).

RNA isolation. Total RNA was isolated from either $100 \mathrm{mg}$ normal epithelia or $100 \mathrm{mg}$ tumor using TRIzol reagent (Invitrogen, Paisley, UK) according to the manufacturer's instructions. Quality of isolated RNA was assessed on a $1 \%$ agarose gel based on the relative abundance of $18 \mathrm{~S}$ and $28 \mathrm{~S}$ subunits of ribosomal RNA. Isolated RNA was quantified using the Nanodrop ND-1000 spectrophotometer (Nanodrop, Wilmington, DE, USA), and stored at $-80^{\circ} \mathrm{C}$ briefly until use.

Microarray experiment. Total RNA $(8 \mu \mathrm{g})$ from 5 patients (tumor and normal epithelial, respectively) was sent for miRNA profiling analysis. miRNA expression profiles were determined at Capital-Bio Corp. (Beijing, China) by using mammalian miRNA arrays (version 3.0), which were designed based on the miRBase release 10.0 and contained 924 probes from humans, mice and rats. The arrays were scanned using a LuxScan $^{\mathrm{TM}}$ laser confocal scanner and the images obtained were analyzed using the LuxScan 3.0 $0^{\mathrm{TM}}$ image analysis package (Capital-Bio Corp., Beijing, China). Raw signal data were normalized by first $\log _{2}$ transformation of signal intensity followed by global variance stabilization normalization (9) of all the arrays within the project.

Quantitative real-time polymerase chain reaction ( $q R T-P C R)$. SYBR Premix Ex Taq (Takara Bio, Dalian, China) was used to quantify mature miRNAs which were differentially expressed in the microarray experiment. cDNA was synthesized by priming with a pool of gene-specific looped primers (RiboBio Biotechnology, Guangzhou, China) including the primers of the miRNA of interest and U6, as an endogenous control. Total RNA $(1 \mu \mathrm{g})$ was reverse transcribed using Takara Reverse Transcription kit (Takara Bio, Inc.) and specific primers for each reverse transcription (RT) reaction. qRT-PCRs were performed in triplicate with SYBR Premix Ex Taq (Takara Bio, Inc.) in $10 \mu \mathrm{l}$ mixtures. qRT-PCRs were conducted by the two-step method on a LightCycler (Roche Diagnostics, Indianapolis, IN, USA). Data for qRT-PCR were analyzed using the comparative $\mathrm{C}_{\mathrm{T}}$ method, which was normalized against the expression of $\mathrm{U} 6$.

Statistical analysis. Differential expression of miRNAs by microarray was determined with the Significance Analysis of Microarray software (Stanford University Labs). A miRNA was determined as differentially expressed if its expression change was $>2$-fold, and it was identified as significantly changed with $\mathrm{q} \leq 5 \%$. A two-tailed Student's t-test was used to compare miRNA expression levels determined by qRT-PCR between supraglottic LSCC tumors with and without lymphatic metastasis.

\section{Results}

In total, the expression of 38 miRNAs was significantly altered between normal laryngeal epithelial and supraglottic LSCC, with 22 being upregulated and 16 being downregulated in tumors $(\mathrm{q} \leq 5 \%)$ (Table I). All 38 miRNAs were human
Table I. Microarray of supraglottic laryngeal squamous cell carcinoma ${ }^{\mathrm{a}}$.

\begin{tabular}{|c|c|c|}
\hline miRNA & $\mathrm{FC}$ & q-value $(\%)$ \\
\hline hsa-miR-1290_st & 9.59 & 0 \\
\hline hsa-miR-196a_st & 3.61 & 0 \\
\hline hsa-miR-455-3p_st & 2.79 & 2.130 \\
\hline hsa-miR-1269_st & 6.96 & 0 \\
\hline hsa-miR-31*_st & 3.45 & 0 \\
\hline hsa-miR-1246_st & 6.04 & 0 \\
\hline hsa-miR-31_st & 3.11 & 0 \\
\hline hsa-miR-21_st & 2.94 & 0 \\
\hline hsa-miR-424*_st & 3.89 & 0 \\
\hline hsa-miR-193b_st & 4.03 & 0 \\
\hline hsa-miR-503_st & 3.44 & 0 \\
\hline hsa-miR-27a*_st & 2.28 & 4.825 \\
\hline hsa-miR-34c-5p_st & 2.52 & 3.502 \\
\hline hsa-miR-196b_st & 2.99 & 0 \\
\hline hsa-miR-339-5p_st & 2.74 & 2.130 \\
\hline hsa-miR-25*_st & 2.41 & 3.502 \\
\hline hsa-miR-222_st & 3.26 & 0 \\
\hline hsa-miR-3178_st & 3.35 & 0 \\
\hline hsa-miR-181b_st & 2.87 & 0 \\
\hline hsa-miR-221-*_st & 2.77 & 2.130 \\
\hline hsa-miR-106b_st & 4.36 & 0 \\
\hline hsa-miR-7_st & 2.27 & 4.825 \\
\hline hsa-miR-125b_st & -3.57 & 0 \\
\hline hsa-miR-3065-5p_st & -2.67 & 2.96 \\
\hline hsa-miR-199b-5p_st & -2.42 & 4.93 \\
\hline hsa-miR-126_st & -2.91 & 1.36 \\
\hline hsa-miR-139-3p_st & -4.23 & 0 \\
\hline hsa-miR-143_st & -2.78 & 2.25 \\
\hline hsa-miR-497_st & -3.26 & 0 \\
\hline hsa-miR-125b-2*_st & -2.68 & 2.96 \\
\hline hsa-miR-617_st & -2.82 & 2.25 \\
\hline hsa-miR-145_st & -2.92 & 1.36 \\
\hline hsa-miR-1224-5p_st & -5.65 & 0 \\
\hline hsa-miR-139-5p_st & -3.67 & 0 \\
\hline hsa-miR-675_st & -3.40 & 0 \\
\hline hsa-miR-195_st & -3.32 & 0 \\
\hline hsa-miR-99a_st & -2.75 & 2.250 \\
\hline hsa-miR-375_st & -7.80 & 0 \\
\hline
\end{tabular}

${ }^{a}$ Annotated human microRNAs differentially expressed in tumors vs. normal epithelial tissues by Significance Analysis of Microarray $(\mathrm{q}<0.05)$. FC, fold change; '-', downregulation.

miRNAs. The expression pattern of the miRNAs listed in Table I with $>2$-fold change is presented as a clustered heat map (Fig. 1), showing a distinct cluster between normal and supraglottic LSCC samples. The most significant aberration was $>9.5$-fold upregulation of miR-1290 and >7.8-fold downregulation of miR-375.

To confirm the microarray results, Taqman qRT-PCR and normalized miRNA expression levels by snRNA U6 were used in 48 supraglottic LSCC patient samples. The three 
Table II. Expression of some miRNAs detected by quantitative real-time polymerase chain reaction ${ }^{\mathrm{a}}$.

\begin{tabular}{|c|c|c|c|c|}
\hline \multirow[b]{2}{*}{ miRNA } & \multicolumn{2}{|c|}{ Tumor (48 cases) } & \multirow[b]{2}{*}{ P-value } & \multirow{2}{*}{$\begin{array}{c}\text { Mucosa } \\
(10 \text { cases })\end{array}$} \\
\hline & Lymphatic metastasis $(17 / 48)$ & No lymphatic metastasis $(31 / 48)$ & & \\
\hline miR-375 & $3.773 \pm 0.104$ & $3.201 \pm 0.553$ & 0.004 & $0.273 \pm 0.024$ \\
\hline miR-139-3P & $2.986 \pm 0.099$ & $2.327 \pm 0.026$ & 0.000 & $0.339 \pm 0.014$ \\
\hline $\operatorname{miR}-1224$ & $2.915 \pm 0.582$ & $2.893 \pm 0.068$ & 0.755 & $0.529 \pm 0.017$ \\
\hline miR-1290 & $0.223 \pm 0.028$ & $0.510 \pm 0.010$ & 0.000 & $3.579 \pm 0.023$ \\
\hline $\operatorname{miR}-1269$ & $0.825 \pm 0.016$ & $0.830 \pm 0.021$ & 0.924 & $3.630 \pm 0.028$ \\
\hline miR-106b & $0.476 \pm 0.038$ & $0.716 \pm 0.320$ & 0.017 & $2.922 \pm 0.056$ \\
\hline
\end{tabular}

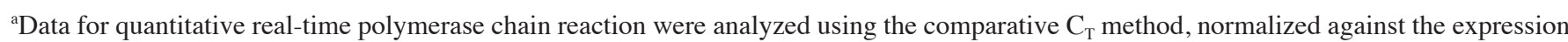
of U6. $\triangle \mathrm{CT}=\mathrm{CT}$ microRNA - CT U6: the higher $\triangle \mathrm{CT}$ is, the lower the microRNA expression.

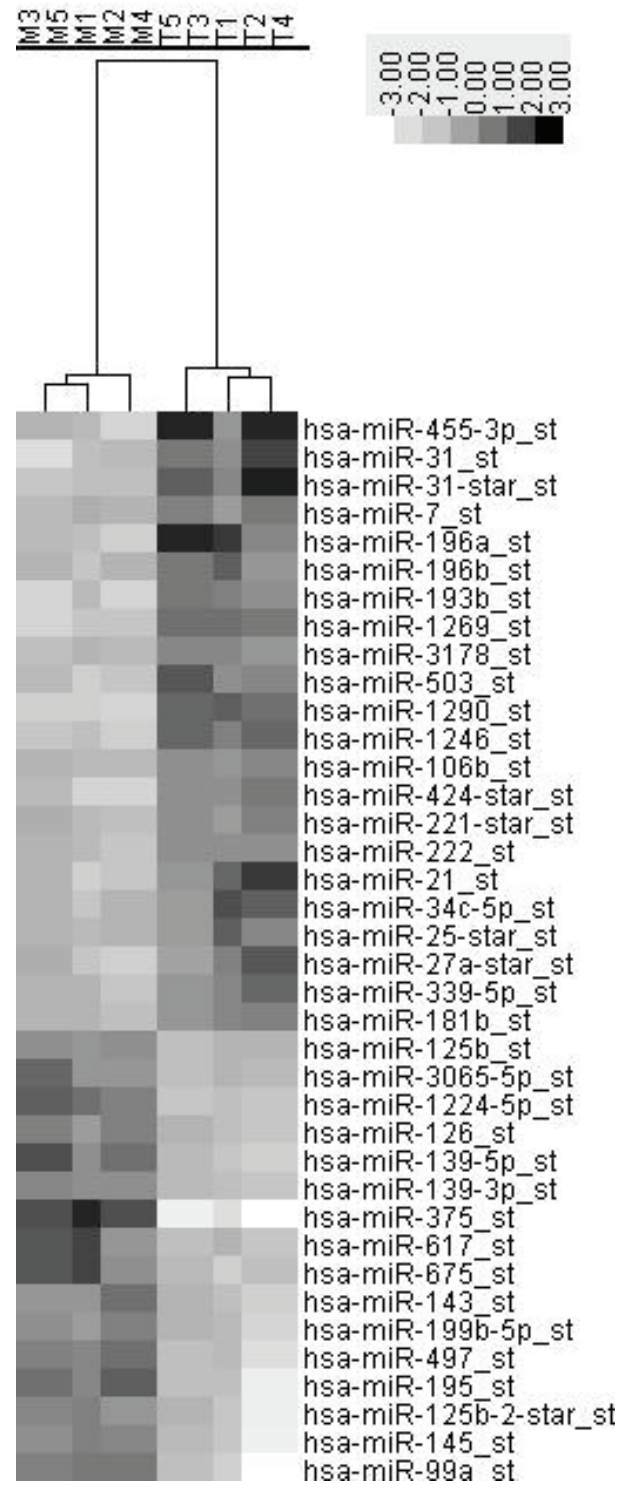

Figure 1. Heat map of the most correlated differentially expressed microRNAS in supraglottic laryngeal squamous cell carcinoma (LSCC). Supervised hierachical clustering of 38 differentially expressed miRNAs with fold changes of $>2$ between five supraglottic LSCC tumors and matched normal mucosa. Tissue samples are shown in the columns and differentially expressed miRNAs are delineated in rows. most up- or downregulated miRNAs were selected for further analysis using qRT-PCR. All the miRNAs tested were reliably confirmed, showing significant differential expression between tumor and mucosa. miR-375, miR-139-3P, miR-1290 and miR-106b showed significantly differential expression between patients with and without lymphatic metastasis (Table II).

\section{Discussion}

The global expression of miRNAs in head and neck squamous cell carcinoma (HNSCC) has recently been reported $(10,11)$. However, HNSCC includes various subtypes, including nasopharyngeal, oropharyngeal, hypopharyngeal, laryngeal and tongue carcinomas. Each subtype is individually characterized in clinic, particularly with regard to metastasis and prognosis, indicating a potential difference in their miRNA expression. Supraglottic LSCC is the most common type of LSCC identified in northeast China (12), and its relatively early lymphatic metastasis and poor prognosis distinguish it from other types of LSCC. To the best of our knowledge, this is the first study regarding miRNA expression profiles in supraglottic LSCC and adjacent normal mucosa. Microarray profiling of >900 miRNAs identified 38 miRNAs that were significantly differentially expressed in tumor tissues compared to normal mucosa, but were not all the same with $\operatorname{HNSCC}(10,11)$. Of the 38 miRNAs, 6 miRNAs were selected for in-depth examination of a larger population of fresh-frozen supraglottic LSCC and normal laryngeal epithelia mucosa by qRT-PCR. Of these 6 miRNAs, 4 had significantly different expression in tumors with and without lymphatic metastasis.

Some of the miRNAs identified as differentially expressed in supraglottic LSCC compared to normal tissues have been characterized in other types of cancer. Overexpression of miR-375 in liver cancer cells decreased cell proliferation, clonogenicity, migration/invasion and induced G1 arrest and apoptosis (13). Additionally, miR-375, which significantly inhibited cancer cell proliferation and invasion in maxillary sinus squamous cell carcinoma, was restored (14). In a recent study, stage and distant metastases revealed differential expression of miR-139-3p in colorectal cancer (15). In the present study, the most significantly downregulated miRNA was miR-375, being underexpressed by 7.8 -fold compared with 
normal tissues, while tumor with lymphatic metastasis had a significantly lower expression compared with tumor without lymphatic metastasis. This result suggests miR-375 may depress the lymphatic metastasis of supraglottic LSCC, such as miR-139-3P. Upregulation of microRNA-1290 impaired cytokinesis and affected the reprogramming of colon cancer cells (16). Overexpression of miR-106b promoted metastasis of hepatocellular carcinoma and correlated with higher tumor grade (17). In the present study miR-106b and miR-1290 had a significantly higher expression in tumor with compared to without lymphatic metastasis, suggesting that interference of the two miRNAs might depress the lymphatic metastasis of supraglottic LSCC. However, although the expression of miR-1224 and miR-1269 were significantly different between tumor and mucosa, their expression showed no significant difference between tumors with and without lymphatic metastasis. This finding indicated that the two miRNAs did not affect metastasis of supraglottic LSCC.

In conclusion, the global miRNA profiling of supraglottic LSCC and attached normal epithelia has demonstrated that $\sim 38$ miRNAs are dysregulated in this disease. miR-375 was most significantly downregulated. As supraglottic LSCC with lymphatic metastasis, miR-375, miR-139-3p, miR-1290 and miR-106b showed a significantly differential expression compared with tumors without lymphatic metastasis. Therefore, understanding of miRNA in supraglottic LSCC is crucial in the development of novel insights for the diagnosis and prognosis of this disease.

\section{Acknowledgements}

This study was supported by the Grants from Education Department of Liaoning Province, China (L2010638).

\section{References}

1. Sieqel R, Naishadham D and Jemal A: Cancer statistics, 2012. CA Cancer J Clin 62: 10-29, 2012.

2. Bussu F, Ranelletti FO, Gessi M, Graziani C, Lanza P, Lauriola L, Paludetti G and Almadori G: Immunohistochemical expression patterns of the HER4 receptors in normal mucosa and in laryngeal squamous cell carcinomas: antioncogenic significance of the HER4 protein in laryngeal squamous cell carcinoma. Laryngoscope 122: 1724-1733, 2012.

3. Bartel DP: MicroRNAs: genomics, biogenesis, mechanism, and function. Cell 116: 281-297, 2004.

4. Du T and Zamore PD: Beginning to understand microRNA function. Cell Res 17: 661-663, 2007.
5. Lu J, Getz G, Miska EA, Alvarez-Saavedra E, Lamb J, Peck D, Sweet-Cordero A, Ebert BL, Mak RH, Ferrando AA, Downing JR, Jacks T, Horvitz HR and Golub TR: MicroRNA expression profiles classify human cancers. Nature 435: 834-838, 2005.

6. Guo Y, Chen Z, Zhang L, Zhou F, Shi S, Feng X, Li B, Meng X, Ma X, Luo M, Shao K, Li N, Qiu B, Mitchelson K, Chenq J and He J: Distinctive microRNA profiles relating to patient survival in esophageal squamous cell carcinoma. Cancer Res 68: 26-33, 2008.

7. Iorio MV, Visone R, Di Leva G, Donati V, Petrocca F, Casalini P. Taccioli C, Volinia S, Liu CG, Alder H, Calin GA, Ménard S and Croce CM: MicroRNA signatures in human ovarian cancer. Cancer Res 67: 8699-8707, 2007.

8. Blenkiron C, Goldstein LD, Thorne NP, Spiteri I, Chin SF, Dunning MJ, Barbosa-Morais NL, Teschendorff AE, Green AR, Ellis IO, Tavare S, Caldas C and Miska EA: MicroRNA expression profiling of human breast cancer identifies new markers of tumor subtype. Genome Biol 8: R214, 2007.

9. Huber W, von Heydebreck A, Sultmann H, Poustka A and Vingron M: Variance stabilization applied to microarray data calibration and to the quantification of differential expression. Bioinformatics 18 (Suppl 1): S96-S104, 2002.

10. Ramdas L, Giri U, Ashorm CL, Coombes KR, EI-Naggar A, Ang KK and Story MD: miRNA expression profiles in head and neck squamous cell carcinoma and adjacent normal tissue. Head Neck 31: 642-654, 2009.

11. Hui AB, Lenarduzzi M, Krushel T, Waldron L, Pintilie M, Shi W, Perez-Ordonez B, Jurisica I, O'Sullivan B, Waldron J, Gullane P, Cummings B and Liu FF: Comprehensive microRNA profiling for head and neck squamous cell carcinomas. Clin Cancer Res 16: 1129-1139, 2010.

12. Fu WN, Shang C, Huang DF, Xu ZM, Sun XH and Sun KL: Average-12.9 chromosome imbalances coupling with 15 differential expression genes possibly involved in the carcinogenesis, progression and metastasis of supraglottic laryngeal squamous cell cancer. Zhonghua Yi Xue Yi Chuan Xue Za Zhi 23: 7-11, 2006 (In Chinese).

13. He XX, Chang Y, Meng FY, Wang MY, Xie QH, Tang F, Li PY, Song YH and Lin JS: MicroRNA-375 targets AEG-1 in hepatocellular carcinoma and suppresses liver cancer cell growth in vitro and in vivo. Oncogene 31: 3357-3369, 2012.

14. Kinoshita T, Nohata N, Yoshino H, Hanazawa T, Kikkawa N, Fujimura L, Chiyomaru T, Kawakami K, Enokida H, Nakagawa M, Okamoto $\mathrm{Y}$ and Seki N: Tumor suppressive microRNA-375 regulates lactate dehydrogenase B in maxillary sinus squamous cell carcinoma. Int J Oncol 40: 185-193, 2012.

15. Chen WC, Lin MS, Ye YL, Gao HJ, Song ZY and Shen XY: MicroRNA expression pattern and its alteration following celecoxib intervention in human colorectal cancer. Exp Ther Med 3: 1039-1048, 2012.

16. Wu J, Ji X, Zhu L, Jiang Q, Wen Z, Xu S, Shao W, Cai J, Du Q, Zhu Y and Mao J: Up-regulation of microRNA-1290 impairs cytokinesis and affects the reprogramming of colon cancer cells. Cancer Lett 329: 155-163, 2013.

17. Yau WL, Lam CS, Ng L, Chow AK, Chan ST, Chan JY, Wo JY, $\mathrm{Ng} \mathrm{KT}$, Man K, Poon RT and Pang RW: Over-expression of miR-106b promotes cell migration and metastasis in hepatocellular carcinoma by activating epithelial-mesenchymal transition process. PLoS One 8: e57882, 2013. 